\title{
Impacto de un curso mediado por la Web 2.0 en el desarrollo profesional de un grupo de futuros docentes de inglés
}

\author{
Impact of a Web 2.0 Mediated Course in the \\ Professional Development of a Group of Future \\ English Teachers
}

Luz Dary Arias Soto ${ }^{1}$

Resumen

Este artículo presenta los resultados de un estudio de investigación desarrollado con estudiantes de licenciatura en inglés de la Universidad Pedagógica Nacional, la cual se fundamentó en constructos teóricos relativos a las competencias de docentes en TIC y desarrollo profesional docente de las TIC en Colombia, enseñanza aprendizaje de lenguas mediados por TIC, y la Web 2.0 aplicada a la enseñanza de la lengua, y que permitió comprobar la eficiencia de herramientas como podcasts, blogs, microblogs y wikis en el desarrollo profesional de un grupo de futuros docentes de inglés en tres aspectos: Tecnológico, pedagógico y lingüístico. La información recogida a través de entrevistas, documentos de los estudiantes publicados en la red, y un pretest y un pos test evidenció la necesidad de mejorar las políticas de desarrollo profesional docente con el fin de formar al profesorado de inglés de bachillerato de forma más acorde con la situación actual respecto a la enseñanza de idiomas mediante el uso de la tecnología, a la vez que permitió comprobar que los participantes del estudio mejoraron su conocimiento y uso de las herramientas, cambiaron su actitud y percepción de las mismas al considerarlas herramientas pedagógicas que cumplen un rol en la educación y mejoraron el dominio de la lengua oral y escrita.

\section{Palabras clave:}

Desarrollo profesional docente, Web 2.0, podcast, blog, microblog, wiki.

\section{Abstract}

This article presents the results of a research study conducted with a group of in training teachers. This research, based on theoretical constructs such as teacher's ICT competences, teacher professional development in ICTs in Colombia, ICT mediated language teaching and learning, and Web 2.0 applied to language teaching and learning, revealed the efficiency of tools such as podcasts, blogs, microblogs and wikis in the professional development of a group of future teachers in three aspects: technologic, pedagogic and linguistic. The analysis of the data collected through interviews, students' documents (blogs, microblogs, and podcasts), and a pretest and a postest evidenced the need to improve the teacher professional development policies of the country so as to educate teachers to meet the demands of a technology mediated learning and teaching language process, and helped determine that the students improved their knowledge and use of the tools, and changed their attitudes and perceptions of the tools. It was also determined that the students improved their command of English both orally and in written form.

\section{Keywords:}

Teacher professional development, Web 2.0, podcast, blog, microblog, wiki.

Artículo recibido el 24 de enero de 2012 y aprobado el 19 de julio de 2012

1 Docente e investigadora Departamento de Lenguas Universidad Pedagógica Nacional, Bogotá, Colombia. Correo electrónico: larias@pedagogica.edu.co 
El desarrollo de la tecnología informática ha ocasionado grandes cambios y progresos no sólo en las diversas áreas del conocimiento, sino también en variados ámbitos de las relaciones entre los seres humanos: lo económico, lo social, lo político, lo cultural y por supuesto, la educación. En este último ámbito ha supuesto un gran paso en la mejora de la enseñanza y aprendizaje de lenguas extranjeras, creando a su vez comunidades de práctica especializadas en la materia y apoyando la investigación lingüística en todas sus áreas.

Así como se ha incrementado el interés a nivel mundial por mejorar el conocimiento y aplicación de las tecnologías de la información y la comunicación (TIC) en la enseñanza, en Colombia se ha otorgado en los últimos diez años gran importancia a la integración de estas al trabajo en el aula, tanto a nivel de bachillerato como a nivel universitario. Por esta razón, el Ministerio de Educación Nacional (MEN, 2008), por medio del Programa Nacional de Nuevas Tecnologías, emplea todos sus esfuerzos en mejorar su política de actuación mediante la formación docentes de todas las áreas. Entre otros objetivos, este ministerio persigue las siguientes metas:

1. Ayudar en la construcción de una infraestructura tecnológica de calidad, para lo cual existe un programa de dotación de instituciones con ordenadores y conectividad.

2. Desarrollar contenidos de calidad a través del Portal Educativo Colombia Aprende, el cual es un espacio de información y conocimiento en el que se producen y se comparten herramientas, contenidos y servicios para la generación de conocimiento en la comunidad educativa del país, para educación preescolar, básica, media y superior.

3. Fomentar el uso y la apropiación de la tecnología, para lo cual se han implementado modelos de desarrollo profesional docente que brindan formación, soporte y acompañamiento a docentes y directivos en el uso de nuevas tecnologías, y definir estándares de calidad para los programas académicos apoyados en el uso de TIC; propiciar redes y comunidades de aprendizaje y evaluar los resultados e impactos de la política.
Sin embargo, la experiencia de trabajar con docentes de colegios públicos de Bogotá, dentro de otros proyectos de investigación en la Universidad Pedagógica Nacional, nos ha mostrado que un gran número de docentes de inglés como lengua extranjera carece de los conocimientos necesarios para enseñar y evaluar mediante la tecnología, en especial Internet, como ha planteado el Ministerio. Tal y como se desprende de los tres objetivos señalados, la tercera línea de acción que mencionamos está relacionada con lograr que los docentes sean capaces de responder a las exigencias de internacionalización y globalización del mundo actual, a través del conocimiento y uso de las TIC. El resultado de esta experiencia es lo que nos llevó a desarrollar esta investigación, cuyo propósito era comprobar la eficiencia de un curso mediado por la Web 2.0 en el desarrollo profesional de un grupo de futuros docentes de lengua inglesa en una universidad pública de Bogotá en tres aspectos: tecnológico, pedagógico y lingüístico.

La pregunta de investigación que se planteó fue:

¿Qué posible impacto puede tener la Web 2.0 en el desarrollo profesional de un grupo de docentes de inglés en formación?

\section{Fundamentación teórica}

\section{Nuevas competencias docentes del profesorado de lengua extranjera}

La inclusión de las TIC como herramientas de la enseñanza y el aprendizaje ha provocado un cambio en el papel que tradicionalmente ha desempeñado el docente a lo largo de la historia. La manera tradicional de enseñar y aprender las lenguas ha cambiado de tal forma que se requiere un docente diestro en el diseño y conocimiento del uso eficiente de la tecnología (Norton y Wiburg, 1998) y de una nueva concepción de su identidad como docente:

Las nuevas modalidades en los procesos de enseñanza y aprendizaje están exigiendo de los docentes un cambio de prácticas, pero también de conocimiento y concepciones acerca de su propio papel como docente, de su propia identidad 
profesional, de su concepción del tiempo y de la tarea profesional. (Marcelo, 2007, p.1)

Si el profesorado no está preparado para este reto, no podrá cumplir con su nuevo rol que implica, entre otros, los siguientes cambios:

- Poseer habilidades tecnológicas para diseñar ambientes de aprendizaje apropiados para el estudiante, teniendo en cuenta que el mundo actual gira alrededor de las TIC y que los estudiantes algunas veces están más preparados en temas de tecnología que los mismos docentes, debido a que la utilizan en su vida diaria para comunicarse con amigos, formar redes sociales, hacer trabajos de investigación, etc.

- Ser facilitador de procesos de aprendizaje y como tal, pasar a ser un tutor que realiza un seguimiento a los procesos de aprendizaje de sus estudiantes y que realimenta su desempeño.

- Posibilitar la creación de espacios para la interacción, dado que las nuevas y diversas formas de comunicación propiciadas por las tecnologías han cambiado la manera como se relacionan los seres humanos a través de herramientas de la red, tanto asincrónicas como sincrónicas.

- Saber adecuar las herramientas, de las cuales los docentes y discentes disponen para sus necesidades y sus propios intereses. Los primeros deben colaborar en el diseño eficiente de estrategias de aprendizaje para sus estudiantes, en las que se integren las tecnologías de tal manera que apoyen el desarrollo cognitivo, lingüístico, académico y afectivo del estudiante (Butler-Pascoe y Wiburg, 2003).

- Motivar al estudiante para que encuentre cierta analogía o correspondencia entre las actividades que realiza en clase y el mundo real, de forma que el aprendizaje sea significativo (Cabré y Gómez de Enterría, 2006).

- Influir en el alumno para que este se comprometa, participe y persista en el logro de sus objetivos.
Impacto de un curso mediado por la web 2.0 en el desarrollo

profesional de un grupo de futuros docentes de inglés

\section{Desarrollo profesional docente de TIC en Colombia}

Los constantes cambios del sistema educativo plantean la necesidad de que los docentes estén preparados para asumir los retos que dichos cambios acarrean y de que, por tanto, las instituciones encargadas de la educación les ofrezcan programas de desarrollo profesional y de actualización que les brinden los conocimientos y las herramientas necesarias para satisfacer las necesidades de los estudiantes, en un sistema donde el conocimiento humano se duplica cada ocho o diez años. Para Díaz-Maggioli (2003), el desarrollo profesional es un proceso de aprendizaje continuo en el cual el docente participa de manera voluntaria con el fin de mejorar sus prácticas de enseñanza y ajustarlas a las necesidades de aprendizaje de sus estudiantes.

Teniendo en cuenta la anterior definición de desarrollo profesional docente, hay algunos aspectos que el profesor debe dominar (Vergara, Hernández y Cárdenas, 2009) y que, por lo tanto, deben ser contemplados en todo proceso de desarrollo profesional, que consideramos, debe llevarse a cabo no solamente con docentes en servicio sino también con docentes en formación, como sucede en este trabajo de investigación. Estos aspectos son:

- Conocimiento disciplinar, es decir de su área de enseñanza, en nuestro caso la lengua inglesa, a la vez que el conocimiento pedagógico necesario para enseñarla.

- Herramientas para construir una identidad profesional y cultural fuerte por ser el docente un agente de cambio social.

- Motivación para desarrollar procesos de investigación como parte de su quehacer diario en el aula.

- Capacidad para reflexionar sobre su desempeño, para autoevaluarse y para evaluar otros individuos que participan del proceso educativo, como son sus colegas y sus estudiantes.

- Habilidades para integrar y hacer uso de los recursos de su contexto.

En este último aspecto, relacionado con los recursos y que atrae toda nuestra atención, debido a la naturaleza de este proyecto de investigación, 
consideramos que más que manejar los recursos con que cuenta el docente a su alrededor, para estar al día en los cambios de la sociedad, este debe conocer los retos de la tecnología en la vida diaria y el aprendizaje y diseñar procesos de instrucción que usen las tecnologías para responder a dichos retos (Norton y Wiburg, 1998, p.13). Por esta razón, todo programa de formación y/o actualización del profesorado debería tener un componente que permita al docente mejorar sus competencias en esta área. Sin embargo, el sistema educativo no prevé una capacitación permanente del cuerpo docente, pese a que el conocimiento humano se duplica cada ocho o diez años. En algunos casos, se hacen cursos aislados sin ningún tipo de seguimiento formal, en otros no se tienen los recursos económicos necesarios para su óptima realización, y en otros, las instituciones encargadas de la formación docente no cuentan con acceso a tecnología actualizada que permita cambiar los métodos de enseñanza (Osín y Huergo, 1999, p.19).

A nivel de estudios de pregrado en Bogotá, de las 12 universidades que ofrecen programas de licenciatura en lengua inglesa, únicamente seis ofrecen algún curso relacionado con alguna herramienta mediada por TIC para la enseñanza del idioma. Los otros seis no contemplan el área de las tecnologías en su malla curricular. Como comentan Caballero, Prada, Vera y Ramírez (2007), se ha reportado un débil impacto de las TIC sobre los programas presenciales de pregrado en las universidades colombianas. Esto quiere decir que no todos los docentes que salen de la universidad a enseñar están capacitados para usar las tecnologías en los procesos de enseñanzaaprendizaje del inglés.

Aunque Caballero y otros (2007, p.54) reconocen el esfuerzo de algunas universidades de Colombia por incorporar las TIC a los programas de formación tanto presencial como virtual, falta todavía mucho por hacer para lograr su vinculación a la educación superior. De ahí la necesidad de que los profesores mejoren su preparación a nivel tecnológico, de forma que puedan utilizar tales herramientas en su labor académica. Esa necesidad se evidencia también en los docentes de lengua extranjera. El estudio realizado por Caballero y otros (2007) sobre competencias de TIC a nivel de Colombia, muestra cómo de 136 experiencias educativas con uso de TIC en la educación preescolar, básica y media del sistema educativo colombiano llevadas a cabo por docentes, solo dos de ellas se referían a inglés o a otra lengua extranjera; todas las demás experiencias se dieron en otras asignaturas como ciencias naturales y matemáticas.

Como se puede ver, es necesario que desde las entidades gubernamentales encargadas de fijar las directrices sobre la formación docente, tales como el Ministerio de Educación, pasando por las instituciones educativas tanto privadas como públicas, hasta llegar a los mismos docentes, se busque dar respuesta a la creciente necesidad de que los maestros se formen en competencias de uso de las TIC para mejorar el aprendizaje de sus estudiantes de lengua extranjera.

El Programa Nacional de Nuevas Tecnologías (MEN, 2008, p.11) plantea como competencia del docente:

Que el aprendizaje que pueden y deben lograr los docentes para apropiar las TIC con un sentido pedagógico, debe ir más allá del manejo básico de herramientas de información y comunicación para apoyar el desarrollo y fortalecimiento de otras competencias básicas decisivas para el desarrollo humano y los aprendizajes significativos que se apropian en el aula y se aplican en la vida.

Esto nos lleva a pensar que el docente no solo debe manejar las competencias básicas de uso del ordenador, de la Internet y de otras herramientas mediadas por las TIC, sino que también debe tener la competencia para decidir cómo integrarlas al proceso de aula de manera más eficiente, siendo capaz de evaluarlas, adaptarlas y usarlas en su propio contexto.

En este mismo documento, el MEN establece diversas competencias que el docente debe adquirir en el manejo de las TIC: técnicas y tecnológicas, pedagógicas, comunicativas, colaborativas y éticas, todas con fines de apropiación personal y apropiación profesional. Sin embargo, no hay directrices específicas para el área de lengua extranjera, lo cual 
nos lleva a corroborar que los docentes de lengua inglesa requieren de cursos de desarrollo que los formen o los actualicen en el uso de las TIC, específicamente en su disciplina. Para los propósitos de esta investigación tendremos en cuenta las competencias técnicas, tecnológicas y pedagógicas para uso profesional, pues nuestro objetivo es ayudar al futuro docente en el manejo de TIC para mejorar su quehacer en el aula y dinamizar el proceso de aprendizaje de sus estudiantes.

\section{Enseñanza-aprendizaje de lenguas mediado por TIC}

Así como las TIC han permeado todos los campos de la ciencia, el conocimiento, la cultura y en general la vida diaria del ser humano, también han llegado a impactar la enseñanza (Norton y Wiburg, 1998) y por lo mismo han cambiado la visión que se tenía del aprendizaje de las diversas disciplinas, entre ellas la enseñanza y el aprendizaje de la lengua. Para esta disciplina surgió CALL (Computer Assisted Language Learning), en español ELAO (enseñanza de lenguas asistida por ordenador), que de acuerdo con Egbert y Petrie (2005, p.4), significa el aprendizaje de la lengua en cualquier contexto con, a través o alrededor de la tecnología del ordenador. El origen de ELAO se remonta hacia los años cincuenta, aunque realmente fue en los sesenta y setenta cuando se implementó, de acuerdo con la teoría conductista del aprendizaje, teoría dominante en aquella época, según la cual el ordenador era considerado como un tutor mecánico que permitía al estudiante hacer ejercicios de repetición (drills) (Warschauer, 1996; Casanovas, 2002).

Con la llegada del enfoque comunicativo para el aprendizaje de las lenguas en los setenta y ochenta, el uso del ordenador en el aprendizaje de la lengua pasó de ser un recurso para aprender formas lingüísticas, a ser un recurso para usar dichas formas en situaciones comunicativas (visión funcional de las formas lingüísticas). Fue Underwood (1984, citado en Warschauer, 1996) quien promovió la idea del uso comunicativo del ordenador; en otras palabras, una perspectiva comunicativa de ELAO. Según él, esta perspectiva:
Impacto de un curso mediado por la web 2.0 en el desarrollo profesional de un grupo de futuros docentes de inglés

Luz Dary Arias Soto

- Se centra más en el uso de las formas que en las formas mismas.

- Enseña la gramática implícitamente y no explícitamente.

- Permite al estudiante y lo motiva a generar un discurso original más que a manipular un discurso prefabricado.

- No juzga ni evalúa todo lo que el estudiante hace ni lo recompensa con mensajes de campanas, luces, u otros.

- Evita decir al estudiante que está equivocado y es flexible con variedad de respuestas.

- Utiliza exclusivamente la lengua extranjera y crea un ambiente en el cual esta se usa de manera natural.

Posteriormente vino una tercera etapa en el desarrollo de ELAO, llamada "integradora" por Warschauer (1996), caracterizada por la llegada de la tecnología multimedia (uso de diversos medios: textos, video, sonido, gráficos, etc.) y de la Internet. Esta etapa se caracteriza porque el aprendizaje de la lengua propende por el uso de la misma en contextos reales y significativos, se busca integrar las diversas habilidades de la lengua y el aprendiz puede conducir su proceso de aprendizaje a su propio ritmo.

Esta gran revolución en la enseñanza-aprendizaje de las lenguas trajo consigo enormes cambios en todos los elementos que constituyen este proceso: el currículo, los entes que toman las decisiones en relación con la educación, el ambiente educativo y, naturalmente, en los docentes y los aprendices. En primer lugar, con la llegada de ELAO se hizo necesaria la adquisición de ordenadores, la construcción de espacios adecuados como salas de autoaprendizaje, espacios con conectividad eficiente, con fácil acceso a diversos medios; asimismo, se hizo necesaria la flexibilización en la organización y los horarios de la escuela, con el fin de facilitar el uso de los espacios y las herramientas (Cunningham, 2000).

Enunciaremos algunos beneficios que Osín y Huergo (1999, p.21) mencionan del aporte computacional a la enseñanza y el aprendizaje. Aunque son beneficios que según estos autores, se evidencian en la educación en general, consideramos que también son susceptibles de evidenciar en la enseñanza y el 
aprendizaje de las lenguas, dada la era de ELAO integradora:

1. Posición activa: mediante la exploración y no por la recepción pasiva de información aportada por el profesor, el estudiante es protagonista de la construcción de su propio conocimiento. Carlson (2004, citado en Barnes, Marateo y Ferris, 2007) habla de los "net geners" o generaciones que han crecido con la Internet, y comenta que ellos mismos son los que deciden sobre el tipo de educación que quieren recibir, o mejor, construir, y de "qué, dónde y cómo aprenden", a diferencia de generaciones anteriores, que no tenían realmente incidencia en la toma de decisiones sobre su educación.

2. Redes de comunicación: la clase no está aislada y se multiplican los recursos de información, se interactúa con otros individuos y con otras comunidades, lo cual permite al estudiante mejorar su desarrollo. Esto coincide con la idea fundamental de Vygotsky (1979), quien explica el desarrollo del ser humano como determinado por la interacción que establece con sus congéneres.

Otras ventajas que hemos observado en nuestra experiencia con ELAO son:

3. Diversidad de ritmos, estilos y estrategias de aprendizaje: el hecho de que las herramientas de TIC sean multimodales ayuda a que los estudiantes mejoren su nivel de lengua de acuerdo con sus estilos de aprendizaje, ya sean visuales, táctiles, auditivos, etc.

4. Inclusión de todas las habilidades y aspectos de la lengua: actualmente, el estudiante hace uso de diversas herramientas informáticas a su alcance para trabajar, de forma relativamente guiada, cualquiera de las competencias básicas de la lengua, así como la pronunciación, la gramática, la morfología, el vocabulario, etc.

5. Mejora de la competencia comunicativa: las aplicaciones tecnológicas encajan perfectamente en lo que Littlewood (1981, citado en Butler-Pascoe y Wiburg, 2003, p.26) establece como herramientas que ayudan al estudiante a reconocer las funciones comunicativas de las formas lingüísticas que se usan, el significado comunicativo en situaciones concretas y el uso de la realimentación para evaluar la transmisión correcta del mensaje que se quiere dar.

6. Aprendizaje y refuerzo: ELAO no solamente permite al estudiante adquirir nuevos conocimientos sobre la lengua, sino que también le permite reforzar los ya adquiridos. Para Pérez las TIC, y especialmente la web, son un entorno constructivista, dado que no solo permiten adquirir conocimientos sino construirlos, puesto que esta es una herramienta centrada en el alumno que le posibilita procesos de reflexión, análisis, síntesis y evaluación.

No obstante los beneficios que las TIC pueden ofrecer para el desarrollo constructivo del conocimiento, Garrison (1993) aclara que estos son potencialidades de las nuevas tecnologías y que, por lo tanto, se debe buscar el mejor uso, dado que ellas se pueden fosilizar y convertirse en "un mero añadido que permita una transmisión de datos más económica y rápida para el consiguiente consumo y adquisición por parte de los estudiantes" (en Bautista, Martínez y Sainz, sin fecha, p.5). Según Bautista y otros, se debe usar las TIC para:
...maximizar las oportunidades de intercambios entre los componentes del proceso de aprendizaje (alumnos, profesores, tutores, materiales) de for- ma que se fomenten las ocasiones para integrar, reconstruir y crear conceptos y significados a tra- vés de actividades en las que el estudiante necesite explicar, elaborar o defender sus posicionamientos ante otros y ante sí mismo.

7. Competencia intercultural: aparte de que ELAO permite al estudiante mejorar su nivel de lengua en relación con las competencias puramente lingüísticas, también le ayuda a adquirir una visión más cercana y real de la cultura de la citada lengua y a mejorar su competencia intercultural.

8. Variedad y autenticidad de recursos: de acuerdo con Norton y Wiburg (1998), las 
tecnologías integradas a las experiencias de aprendizaje ayudan al estudiante a tomar decisiones, a desarrollar procesos de investigación, a resolver problemas reales y a participar en comunidades de conocimiento, todo debido a la diversidad de herramientas mediadas por el ordenador.

9. Trabajo colaborativo y cooperativo, de manera sincrónica o asincrónica: los usuarios, en este caso los estudiantes, pueden interactuar de manera independiente del tiempo, del lugar, etc., lo cual permite superar problemas de espacio en las instituciones y dificultades de horarios en los diversos individuos que forman parte del proceso enseñanza-aprendizaje.

10. Gran cantidad de input (información que se recibe): dada la enorme cantidad y variedad de información tanto oral como escrita que se encuentra en la red, en nuestro caso en inglés, el estudiante recibe input constante, a tal punto que podríamos hablar de una inmersión virtual en la lengua extranjera, siguiendo las palabras de Chappelle (2003, p.36).

11. Satisfacción de las necesidades afectivas de los estudiantes: para Butler-Pascoe (2003, en Butler-Pascoe y Wiburg, 2003, p.2), mediante el uso de la red el estudiante se puede sentir parte de una comunidad más grande que la del aula, puede corregir sus errores sin pasar por la pena que implica ser corregido en la clase presencial; siente más seguridad participando en la comunicación por medio de la red que hablando frente a frente con sus compañeros de clase y su autoestima mejora al producir textos publicables en la web y al lograr comunicarse con otras personas.

Cantallops, González y Yáñez (1999) afirman que el medio telemático favorece el aprendizaje autónomo, incita a la participación del estudiante más introvertido y permite un seguimiento más individualizado y personal, contribuyendo así a reforzar la autoestima del estudiante. Esto ayuda a que el estudiante disponga en todo momento de una retroalimentación inmediata de lo producido, respeta su ritmo de aprendizaje y contribuye a mantener su motivación. Warschauer (1996) reconoce así la influencia positiva de ELAO en la motivación del aprendiz de la lengua, puesto que este se siente motivado al ser consciente del propósito de las actividades a nivel social y cultural (Warschauer, 2000, p.52), lo cual se logra con la tecnología.

\section{La Web 2.0 aplicada a la enseñanza de idiomas}

Anderson (2007) presenta la Web 2.0 como algo más que un espacio de información global, algo que va más allá de ser una red social para convertirse en una especie de "fábrica social" que se construye ante nuestros ojos a través de la colaboración, la construcción y el trabajo en comunidad. El carácter de ser un espacio en el que el usuario puede editar, agregar información, modificarla, etc., la hace una versión más avanzada de la Web 1.0, pues de acuerdo con Anderson (2007), la Web 2.0 no es un producto opuesto a la Web 1.0, sino una versión más desarrollada de la misma. Warschauer y Grimes (2007, p.2), al referirse a la distinción entre la Web 1.0 y la Web 2.0, afirman que la diferencia entre ellas está en la publicación y la participación. La primera permitía al usuario publicar contenido que al final terminaba aislado en la red, mientras que la segunda permite formas interactivas de publicación de contenido textual y multimodal, de creación de y participación en redes sociales a través de blogs, wikis y otros sitios, plataformas, etc.

Veamos cuales son algunas de las características y, al mismo tiempo, ventajas de la Web 2.0:

- Es una plataforma fácil de usar: como comentan Ruipérez, García, Castrillo y Román (2008), con solo pulsar el ratón es suficiente, pues no se necesitan grandes conocimientos de informática.

- Potencia y ofrece nuevos escenarios de aprendizaje, dado que se puede tener acceso a centros de investigación, universidades, comunidades de conocimiento, y permite 
que haya intercambio de conocimientos con docentes, otros estudiantes y otros usuarios de la red.

- Facilita, a través de la interacción sincrónica o asincrónica que se da entre los estudiantes, procesos de negociación de significado, de atención a aspectos formales de la lengua y de co-construcción del significado (Chappelle, 2003, p.57).

- Favorece la comunicación y la adquisición de conocimientos e información debido a que se tiene fácil acceso a todo tipo de documentos, ya sean de texto, de video, de audio, fotográficos, etc., y entre usuarios de lugares apartados, lo cual rompe las barreras que se pueden dar debido a las distancias.

- Las herramientas cooperativas de la Web 2.0 fomentan la atención en los aspectos del uso de la lengua, que van desde la selección de léxico y la precisión sintáctica hasta el estilo retórico para lograr cohesión textual (Guzzo, $\sin$ fecha).

A continuación describiremos las herramientas de la Web 2.0 que utilizaremos en esta propuesta:

\section{Blogs}

Los blogs, también llamados weblogs, son sitios web o parte de un sitio web con entradas de comentarios, descripciones de hechos, gráficos o videos, etc., publicados en la red por usuarios de la misma. Además, son sitios que permiten la interacción entre su autor y otros internautas, que para nuestro caso pueden ser el estudiante, el docente, otros estudiantes de la misma lengua, hablantes nativos, hablantes no nativos, etc.

El rápido avance en la facilidad de publicación de un blog permitió que otras personas, no solamente expertas en el diseño de páginas web, pudieran acceder a este recurso, razón por la cual la comunidad de blogueros se disparó en cuanto a la cantidad de blogs que existen en la red y también en cuanto a los campos en los que se usan los blogs actualmente: periodismo, guerra, medicina, política, negocios, etc., y, por supuesto, la educación. En relación con este último tema, que es el que nos atañe, los blogs se pueden utilizar de diversas maneras (De la Torre, 2006) a través de:

1. Blogs de asignatura, en los cuales el profesor va publicando noticias sobre la misma, pidiendo comentarios de sus alumnos a algún texto, haciendo propuestas de actividades, informando sobre calendarios, etc. De acuerdo con Campbell (2003) los blogs de clase o asignatura pueden ser utilizados en clases de conversación para publicar mensajes, imágenes, temas de discusión; en clases de diversos niveles para actividades de aprendizaje de la lengua basado en proyectos; como espacio virtual para intercambio comunicativo entre usuarios de diferentes países; y para clases de escritura y de lectura.

2. Blogs individuales de alumnos en los que se les pide la escritura de entradas periódicas, a las que se les hace seguimiento y son apoyadas no solo en los aspectos relacionados con la temática o contenidos tratados, sino también sobre asuntos relacionados con derechos de autor, normas de estilo, citación de fuentes, etc.

3. Blogs grupales de alumnos en los que, de forma colectiva a modo de equipo de redacción, tendrán que publicar entradas relacionadas con las temáticas, estilos y procedimientos establecidos. Este tipo de blog promueve el aprendizaje colaborativo y el aprendizaje cooperativo.

Los blogs brindan multitud de beneficios en la educación; tal como señala Richardson (2006), fomentan el pensamiento crítico y analítico, así como el pensamiento analógico. Además, ofrecen el acceso a información variada y de calidad, y promueven la interacción social. En la misma línea de pensamiento, Jordano (2004) afirma que los blogs son útiles en la enseñanza y aprendizaje de la lengua, dado que se pueden utilizar con estudiantes de niveles de lengua intermedios y superiores, y son motivantes para estudiantes de niveles avanzados; potencian el pensamiento crítico en la L2 y permiten que el 
estudiante incluso llegue a expresar sus sentimientos en una lengua diferente a la suya propia.

\section{Podcasts}

El podcast, término resultante de la combinación de las palabras ipod ${ }^{1}$ y broadcasting (emisión) (Stanley, 2006), es una grabación en audio, generalmente en formato MP3, de entrevistas, conversaciones, ponencias, monólogos, etc., creada para su distribución en Internet y para ser reproducida en lectores de sonido portátiles y en ordenadores. Por ser herramientas de audio y video, los podcasts resultan de gran utilidad en el aula de lengua extranjera para desarrollar la competencia oral receptiva así como para mejorar la expresión oral. Cuando el estudiante diseña y pone su grabación (o podcast) en la red, se espera que lo haga con un buen nivel de manejo de la lengua, según su dominio de la misma. Estos pueden ser usados para realizar diversas actividades como evaluar proyectos de los estudiantes, exámenes, presentaciones en video, entrevistas de estudiantes por parejas, dictados, discusiones de mesa redonda, presentación de invitados expertos en alguna materia, etc. (Abdous, Camarena y Facer, 2009). Los podcasts brindan al estudiante la oportunidad de recibir input en la lengua meta sobre temas variados según sus intereses personales.

En un estudio llevado a cabo por Edirisingha, Nie, Rizzi y Rothwell (2007) se concluyó que los podcasts favorecen el aprendizaje debido a tres rasgos que los caracterizan: la posibilidad de elección y su flexibilidad en cuanto a tiempo, contenidos, origen de la información, entre otros; la posibilidad de aprendizaje informal y el conocimiento tácito al cual se puede tener acceso a través de la discusión. Otros beneficios del uso de podcasts en el aula de lengua extranjera son los reportados por Chacón y Pérez (2009), quienes afirman que la incorporación de podcasts al proceso de aprendizaje de la lengua inglesa promueve la cooperación y la interacción, contribuye al desarrollo de las habilidades de producción oral y comprensión auditiva, desarrolla la autonomía y la autorregulación

1 Reproductor de música digital de tamaño pequeño que consta de un disco duro. del aprendizaje, estimula la colaboración y cooperación entre los aprendices de la lengua y facilita el desarrollo de las habilidades lingüísticas.

\section{Wikis}

El término wiki, de origen hawaiano, significa "rápido" y se refiere a un tipo de entorno colaborativo en el cual los usuarios pueden participar en la modificación, creación o eliminación de una página y de manera colectiva construir su aprendizaje. Según Richardson (2006, p.8) una wiki es un espacio en la red en el cual cualquier persona puede agregar contenido o editar el que ya ha sido publicado.

Las wikis, que se editan de forma colectiva incluyendo textos, enlaces, fotos, información, han ido adquiriendo un valor cada vez más significativo en el sistema educativo actual a todos los niveles, ya que favorecen el aprendizaje colaborativo y la interacción, promueven la negociación y la retroalimentación entre usuarios (Cabezas, 2008). En un estudio llevado a cabo por Lund (2008), en el cual se quería indagar el impacto de las wikis en el aula de clase de lengua extranjera, se concluyó que el trabajo realizado evidenció la zona de desarrollo próximo $(\mathrm{ZDP})^{2}$, pues los estudiantes participaron de manera colectiva en el desarrollo de las tareas y, por tanto, en la construcción colectiva del conocimiento. A propósito de la construcción social del conocimiento, en otro estudio, realizado por Gimeno y García (2009), se concluyó que las wikis son herramientas que permiten la enseñanza y la tutoría entre pares, particularmente para la creación de textos escritos a través del trabajo colaborativo, lo cual, a su vez, hace que el estudiante sea más consciente del proceso de la construcción textual. Asimismo, se estableció que dan al estudiante la posibilidad de participar en tres procesos fundamentales para su creación que son a la vez procesos que se dan en el aprendizaje de lengua extranjera: input, output (producción de información) y retroalimentación, tal como se puede ver en la siguiente figura:

2 “Distancia entre el nivel real de desarrollo determinado por la capacidad de resolver independientemente un problema y el nivel de desarrollo potencial, determinado a través de la resolución de un problema bajo la guía de un adulto o en colaboración con otro compañero más capaz" (Vigotsky, 1979, p.133). 
Figura 1. Proceso cognitivo del estudiante-participante en una wiki. (Davidson, 2008; Xiao y Lucking, 2008, en Gimeno y García, 2009, p. 11).

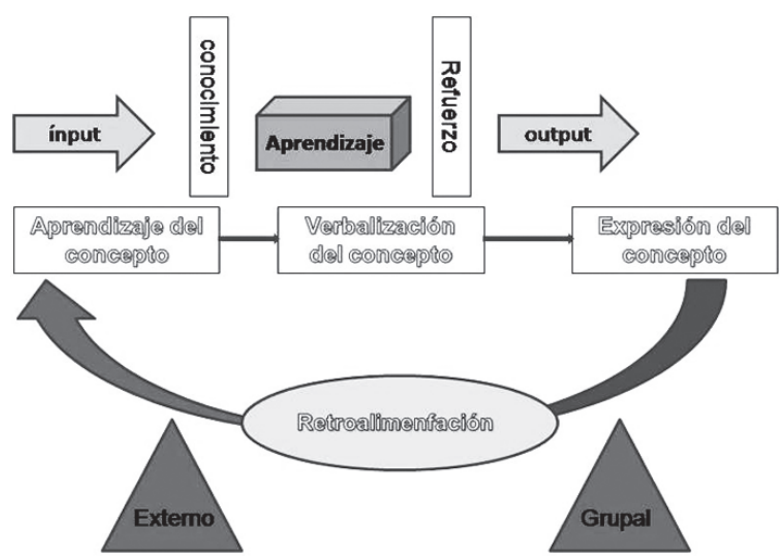

En el trabajo con wikis, el estudiante recibe información, internaliza el concepto o aspecto de la lengua, recibe realimentación por parte de su profesor o de sus compañeros, lo cual se convierte a su vez en input y luego exterioriza ese conocimiento, el cual se convierte en output.

West y West (2009) sugieren la utilización de wikis para diversos propósitos, tales como construcción de conocimiento, desarrollo de pensamiento crítico y aplicación contextual. Este último propósito tiene que ver con la aplicación de conocimientos y habilidades en otros contextos o situaciones problemáticas por parte del estudiante. También, consideramos, se debe tener en cuenta que para el desarrollo de las habilidades de expresión escrita y comprensión lectora, las wikis son herramientas útiles, pues el estudiante puede hacer procesos de lectura simples o complejos y, así mismo, escribir de manera colaborativa. Coincidimos con West y West (2009), pues como ellos afirman, el hecho de brindar al estudiante experiencias de escritura colaborativa estructuradas, puede cerrar la brecha entre la comunicación social y la habilidad para escribir con claridad, lo cual puede, a su vez, ejercer una influencia en el trabajo, en el estudio y en la sociedad.

No hay mucha información relacionada con wikis y su incidencia en el mejoramiento de la lengua extranjera, pero sí sobre otros aspectos como el desarrollo de la autonomía y del trabajo colaborativo a través del uso de dicha herramienta. Un ejemplo de su uso para el desarrollo de la autonomía y el trabajo colaborativo es la experiencia llevada a cabo por Kessler y Bikowski (2010), quienes concluyeron que el trabajo con wikis evidenció que los estudiantes aumentaron su motivación para interactuar con sus compañeros en una relación de igualdad y, al mismo tiempo, aumentaron su interés por participar en lo que otros escribían. Además, en esa experiencia los estudiantes demostraron su habilidad para usar la lengua con el fin de contribuir a la edición de la wiki, como miembros de un grupo, pero también, y de manera individual, recogieron información, evaluaron y compartieron contenidos para el beneficio del grupo, además de llevar a cabo ejercicios de adición y supresión de información, así como de aclaración y síntesis.

\section{Microblogging: Twitter}

El microblogging es un servicio que permite publicar mensajes breves, generalmente de solo texto. Una de las posibilidades de este servicio es Twitter, herramienta basada en la Web 2.0 que consiste en una red social en la que los usuarios tienen la posibilidad de leer y de escribir textos de máximo 140 caracteres, llamados trinos (tweets), para establecer comunicación con otros usuarios.

De acuerdo con Stevens (2008), Twitter tiene algunas ventajas sobre algunas herramientas como los blogs, tales como que se tiene respuesta casi inmediata a un interrogante o comentario que se hace. A través de esta herramienta la conversación es más interactiva que con los blogs y la información es más personal. Por ser textos tan cortos, su escritura exige que el usuario, y en este caso el estudiante, no cometa errores de gramática o de ortografía para que su mensaje sea coherente, conciso y claro y, por consiguiente, haya menos probabilidad de que los seguidores o followers malinterpreten el mensaje que se quiere transmitir. Por este motivo, el estudiante debe hacer muy buen uso de lengua extranjera, lo cual le ayudará a mejorar su dominio de la misma.

Dave (2008) ha utilizado Twitter como herramienta de aprendizaje y práctica de la lengua de diversas maneras: para conversación en clase, para crear comunidad, como herramienta de búsqueda de palabras para aprender cómo se usan, o buscar un evento, un nombre propio, etc. Eso le ha permitido 
evidenciar que los estudiantes se sienten motivados para hablar de diversos temas dentro y fuera de la clase, han incrementado el sentido de pertenencia a un grupo y han mejorado su conocimiento y uso de la gramática. Por su parte, Borau, Ullrich, Feng y Shen $(2009$, p.82) afirman que Twitter brinda oportunidades de práctica de la competencia sociolingüística, dado que el usuario debe tener la habilidad de usar la lengua y entenderla de manera apropiada de acuerdo con el contexto, el tema y la relación que existe entre él y los individuos con los cuales se comunica. Asimismo, reportan que la interacción con hablantes nativos de una lengua por medio de Twitter ayuda a mejorar la conciencia cultural y, por ende, la competencia intercultural del estudiante.

En Colombia se han llevado a cabo numerosos y variados proyectos de inclusión de herramientas mediadas por TIC en los procesos de enseñanzaaprendizaje de las lenguas extranjeras, especialmente de inglés. Sin embargo, muy pocos dan cuenta del uso de herramientas de la Web 2.0, tales como podcasts, weblogs, microblogging o wikis. Un ejemplo de ello es el trabajo realizado por Quintero (2008), en el cual la autora reporta los resultados de un proyecto de investigación-acción llevado a cabo con estudiantes de primer año de licenciatura de inglés en una universidad pública de Bogotá. La comunicación de los estudiantes, colombianos y canadienses, a través de un blog personal, un blog grupal y un blog de debates evidenció que:

... la escritura puede desarrollarse cuando los estudiantes se sienten parte de una comunidad con la cual interactúan y comparten intereses y objetivos de aprendizaje de lengua similares, mediados por la tecnología... a través de la escritura en blogs los estudiantes no solo desarrollan su escritura en EFL, sino que también tienen la oportunidad de proyectar su propia imagen... (p.7).

Otro trabajo es la investigación desarrollada por Cantor (2009, p.119) en el programa ALEX virtual de la Universidad Nacional de Colombia. Este trabajo, llevado a cabo bajo la modalidad de aprendizaje mixto (blended learning) y que usó los foros de discusión como herramienta principal para complementar el trabajo presencial, permitió a Cantor concluir que los foros de discusión promovieron la autonomía, aumentaron el sentido de la responsabilidad e incentivaron a los estudiantes a hacer su mejor esfuerzo para comunicarse de manera eficiente, además de motivarlos para tomar el control de su propio aprendizaje. Cantor sugiere, a raíz de esta investigación, que se les dé importancia a las herramientas asincrónicas de la red, dado que estas son mediadoras en la interacción docenteestudiante, estudiante-estudiante y promueven la autonomía, la interacción y el trabajo colaborativo, mediante la discusión, la negociación de significado y la argumentación.

Vale la pena hacer énfasis en los procesos mencionados anteriormente, dado que son procesos complejos de pensamiento que se desarrollan a través del estudio de la lengua con herramientas mediadas por TIC. Al respecto se puede afirmar que las herramientas como podcasts, blogs, microblogs $\mathrm{y}$ wikis, pueden ser clasificadas como herramientas de pensamiento (mindtools), de acuerdo a la descripción de Jonassen (1996), puesto que ayudan al estudiante a desarrollar el pensamiento crítico, a organizar y representar el conocimiento en lugar de recordar lo que el docente explica; promueven el pensamiento reflexivo y la construcción del conocimiento, hacen al estudiante responsable de su propio aprendizaje y facilitan el aprendizaje significativo.

\section{Metodología}

Los docentes siempre nos vemos enfrentados a situaciones problemáticas que nos exigen actuar para solucionarlas o para atenuarlas y, de esta manera, ayudar a mejorar nuestro contexto educativo. La investigación-acción, escogida como metodología para este trabajo, es una de las metodologías que nos ofrece posibilidades a los docentes de diversas disciplinas de buscar formas de mejorar los procesos de enseñanza y aprendizaje, dentro del aula y fuera de ella, por medio de la resolución de problemas de forma práctica y eficaz (Burns, 2010). Este método de investigación se caracteriza por ser un método evaluativo y reflexivo que ayuda a resolver problemas para mejorar una situación determinada, por integrar la teoría con la práctica. 
Por otra parte, esta investigación es de tipo mixto, dado que una parte del estudio es cuantitativa y otra es cualitativa. Cuantitativa porque en una parte del análisis de los datos es necesario cuantificar algunas frecuencias, y cualitativa considerando que, como afirma Burns (2003), los enfoques cualitativos buscan hacer descripciones e interpretaciones en un contexto social natural, y como Bloomberg y Volpe (2008, p.7) lo expresan, la investigación cualitativa es apropiada para lograr el entendimiento profundo de un contexto social o una actividad, tal como estos son vistos desde la perspectiva de los participantes de la investigación.

\section{Participantes}

La población fue conformada por un grupo de 18 estudiantes entre las edades de 18 y 25 años, de sexto semestre del programa de Licenciatura en Educación Básica con Énfasis en Humanidades: español e inglés, de la Universidad Pedagógica Nacional. Esta, como su nombre lo indica, es una institución cuya misión es exclusivamente formar profesores en diversas áreas del conocimiento, entre ellas la lengua inglesa, la lengua francesa y el castellano como lengua propia. Este grupo de estudiantes cursó Competencias en Lengua Inglesa, mediante la cual se pretende que el estudiante mejore su dominio de la lengua en todas sus competencias comunicativas.

\section{Instrumentos para la recolección de datos}

\section{Entrevistas}

La entrevista es uno de los instrumentos escogidos para esta investigación, dado que se buscaba que los participantes pudieran expresar, mediante la interacción con el investigador, su interpretación de la situación en estudio. Es decir, se buscaba que el estudiante pudiera expresar más de lo que podría manifestar mediante un instrumento más cerrado, como la encuesta, y que participara en la construcción de la información mediante el diálogo intersubjetivo con el entrevistador. El pilotaje de la entrevista final se hizo con cinco estudiantes de un grupo con similares características y que tomaban el mismo curso en un horario diferente. Esto permitió agregar algunas preguntas que permitirían profundizar en varios aspectos.

\section{Pretest y postest}

Aunque el diseño de un pretest y de un postest de un solo grupo puede ser considerado un "mal ejemplo" como instrumento de recolección de datos, puesto que atenta contra la validez de un estudio (Campbell y Stanley, 2001, p.20), dado que puede haber otros acontecimientos ajenos a la intervención del investigador que pueden determinar cambios en la conducta del grupo en estudio, hemos tenido en cuenta la información de un pretest y un postest por considerar que los datos recogidos en ellos nos pueden ayudar a triangular los datos de las entrevistas hechas a los estudiantes.

Antes de iniciar la implementación del curso apoyado en herramientas de la Web 2.0, los estudiantes desarrollaron dos actividades, consideradas como pretest oral y pretest escrito, las cuales nos ayudaron a examinar cuáles eran los errores más comunes que ellos cometían al expresarse verbalmente y en forma escrita, para luego comparar con el postest y ver si mejoraron el manejo de la lengua escrita y oral después de la implementación. La actividad escrita consistió en escribir un ensayo corto, máximo de cinco párrafos, en el cual los estudiantes debían hacer una presentación sobre cómo se veían en cinco años, después de haber terminado su carrera y haber comenzado a trabajar. Para esta actividad, los estudiantes no contaron con ninguna ayuda, ni de parte del profesor ni de sus compañeros, ni tampoco de diccionarios u otras herramientas, ya que se quería verificar el nivel de lengua con el que los estudiantes llegaron al curso.

Para la actividad oral los estudiantes debían organizarse en grupos de tres integrantes, escoger una actividad de grupo tal como mesa redonda, debate o panel y escoger un moderador y un tema controvertido para la discusión. Los estudiantes debían investigar sobre el tema y preparar sus intervenciones, para lo cual se les dio dos días. 
En la presentación misma de la actividad los estudiantes debían hablar espontáneamente, sin leer las notas, y estableciendo comunicación con sus compañeros a medida que se iba dando la discusión.

\section{Documentos de los estudiantes}

Los podcasts, blogs y microblogs de los estudiantes fueron utilizados con el fin de recoger datos que permitieran triangular la información tanto de los tests como de las entrevistas, pues en ellos los estudiantes pudieron describir sus acciones individuales, experiencias y percepciones (Merriam, 2000), los cuales nos permitieron responder la pregunta de investigación.

\section{Rol del investigador}

El investigador cumplió el rol de participante observador: como participante, fue docente del grupo que conforma la población del estudio. El investigador implementó el uso de algunos recursos de la Web 2.0 como complemento para el aprendizaje de la lengua, fue responsable de la práctica pedagógica y participó de todas las actividades planeadas. Como observador, el investigador pudo obtener una descripción detallada y completa de lo que sucedía en el estudio investigativo, mediante la observación del proceso de aprendizaje de los estudiantes y la recogida de datos.

\section{Triangulación de la información}

"La triangulación es una de las maneras más comunes de lograr la validez en un proceso de investigación" (Burns, 2003, p.163). Aunque existen diversas clases de triangulación, para nuestros propósitos decidimos utilizar la triangulación metodológica, recurriendo a la utilización de varios instrumentos de recolección de datos para corroborar la información que se recogió (Denzin, 1978, citado en Freeman, 1998), tales como entrevistas, pretests, postests y documentos publicados en la web.

\section{Implementación de la estrategia}

Con el fin de resolver la pregunta de investigación y de alcanzar los objetivos planteados en esta in- vestigación, se planeó desarrollar la asignatura de Competencias en Lengua Inglesa a través del uso de algunas herramientas de TIC como recursos complementarios para el desarrollo del programa, dado que la Universidad Pedagógica Nacional todavía no ha integrado el uso de las tecnologías de manera obligatoria a ninguno de los cursos ofrecidos por el Departamento de Lenguas. Se escogió esta metodología debido a que el propósito del estudio era analizar, diseñar e implementar un curso complementado con herramientas de la Web 2.0, en el que se esperaba que los estudiantes las usaran como recurso para complementar -no como herramienta única del curso- el trabajo de aula como estudiantes de inglés, aprendiera a manejar las herramientas con fines pedagógicos para usarlas en su futuro quehacer como docentes y mejoraran su nivel de lengua. Puesto que se buscaba analizar los efectos de un curso apoyado en la Web 2.0 en el desarrollo profesional de un grupo de futuros docentes de inglés, varias herramientas de la Web 2.0 sirvieron a los propósitos del estudio: podcasts, microblogging, blogging y wikis, herramientas que fueron utilizadas de la siguiente manera durante los tres meses que tomó esta implementación.

Durante las dos primeras semanas, la docente investigadora presentó el programa de la asignatura y ofreció a los estudiantes información sobre el apoyo del curso en actividades mediadas por algunas herramientas de TIC. Se informó a los estudiantes sobre los propósitos de este trabajo de investigación, en qué consistiría su participación y se les aclaró que en ningún momento se eliminarían contenidos del programa para cumplir con esta implementación, sino que, por el contrario, se tratarían los mismos temas pero con recursos diferentes, como son las herramientas de la Web 2.0. Los estudiantes se mostraron bastante entusiasmados y aceptaron participar en el proyecto de manera voluntaria.

Antes de iniciar la implementación, los estudiantes investigaron de forma autónoma sobre la Web 2.0 para su posterior discusión en clase, de forma que todos tuvieran información casi homogénea sobre el tema. Es importante destacar que antes del trabajo con estas herramientas, se estudió con de- 
talle cada una de ellas en lo relativo a su aplicación en el aprendizaje de lenguas, publicación, edición, personalización, etc. A continuación se inició la implementación con los podcasts, se siguió con microblogging, posteriormente con los blogs y finalmente con las wikis.

\section{Trabajo con podcasts}

Una vez se aclararon las generalidades de la herramienta, los estudiantes tuvieron la oportunidad de escuchar un podcast publicado por la investigadora en la red, sobre la competencia intercultural y la enseñanza de la cultura en el proceso de enseñanza y de aprendizaje de una lengua extranjera (http://luzdarias.podomatic.com/). Seguidamente, los estudiantes debatieron en grupos durante una de las clases. Con base en lo escuchado, los estudiantes hicieron una actividad de expresión oral. Luego se invitó a los estudiantes a escuchar algunos podcasts publicados en la red sobre diversos temas sociales, deportivos, de historia, así como sobre la enseñanza del inglés y de la cultura. Ellos podían escuchar los podcasts que quisieran, según las temáticas de su interés, pero sin salirse de los temas programados para estudiar en la asignatura, teniendo en cuenta que esta giraba en torno a diversas temáticas. En la siguiente sesión los estudiantes se reunieron en grupos y contaron a sus compañeros con sus propias palabras, en inglés, lo que habían escuchado.

Después de este momento, en el cual los estudiantes escucharon podcasts publicados por otras personas, se pasó a la etapa de diseño y publicación de sus propios podcasts, para lo cual decidieron centrarse en un solo tema. Para ello los estudiantes tomaron un tiempo para la redacción de un texto, para su lectura en voz alta ante algunos de sus compañeros, quienes les dieron retroalimentación, y para la lectura ante la investigadora, quien también les hizo sugerencias sobre pronunciación y entonación. Finalmente, los estudiantes publicaron sus podcasts, ilustrándolos con alguna imagen, y se escucharon unos a otros con el fin de conocer lo que sus compañeros opinaban sobre temas como el rol de la tecnología en el aprendizaje de la lengua y el rol del ordenador en el desarrollo del pensamiento crítico en lengua extranjera.

\section{Implementación de microblogging (Twitter)}

Después de indagar un poco sobre el funcionamiento de la herramienta, los estudiantes accedieron a la cuenta de la docente en Twitter para leer la pregunta que ella hacía sobre la sustitución o no del docente por parte del ordenador y dar su opinión al respecto. La actividad siguiente consistía en hacer comentarios sobre la pregunta, leyendo los comentarios de cuatro de sus compañeros. Por limitaciones de tiempo no era recomendable pedir a los estudiantes que hicieran comentarios a todos sus compañeros de clase. Algunos estudiantes escribieron solo un comentario, pero otros escribieron en varias oportunidades. Esta actividad motivó a algunos a abrir su propia cuenta en Twitter y a invitar a sus compañeros a participar en esta forma de comunicación. Con esta herramienta, los estudiantes tuvieron la oportunidad de comunicarse mediante la composición de textos cortos que fueron redactados, leídos, comentados y corregidos (Chappelle, 2003, p. 63). Se trataron temas especialmente de tecnologías, dado que los estudiantes se entusiasmaron al respecto.

\section{Implementación de los blogs}

Para los propósitos de esta investigación se siguieron algunas de las fases que Jordano (2009) plantea: el profesor escribe y los estudiantes responden, tanto a la publicación del profesor como a lo que los compañeros de clase han escrito; el profesor y los estudiantes escriben y los estudiantes conducen su propio blog.Al igual que con todas las herramientas de la implementación, los estudiantes tuvieron un primer contacto con los blogs con el fin de saber más sobre posibilidades que estos ofrecen para el aprendizaje de lenguas y sus formas de publicación. Posteriormente se procedió a hacer la lectura del blog de clase diseñado y subido a la red por la docente investigadora.

Luego se hizo un trabajo individual en el que los estudiantes redactaron sus comentarios a los dos textos en discusión, El papel del docente en la era de TIC y El papel del estudiante en la era de TIC, respectivamente. Los comentarios escritos por los estudiantes permitieron ver si habían comprendido los dos textos publicados. Finalmente, se dio una 
cadena de comentarios en la que los estudiantes daban sus opiniones sobre los textos publicados por la docente.

\section{Implementación de las wikis}

Tras la primera toma de contacto con la herramienta, dos de los estudiantes dieron a conocer el trabajo que ellos habían elaborado con la misma en una asignatura tomada el semestre anterior, e ilustraron cómo de ella se podía hacer una herramienta al servicio de la enseñanza y el aprendizaje no solo de las lenguas, sino de otras disciplinas. A continuación, durante una sesión de clase, se discutió sobre el tema alrededor del cual giraría toda la información que se escribiría, de tal forma que fuera como un sitio en Internet correspondiente a la asignatura. Se creó una lista de temas en la pizarra y se llegó a la conclusión de que todos los textos que se subieran a la página de Internet debían estar relacionados con temas culturales, razón por la cual se le dio el nombre de www.culturalviews. wikispaces.com. A la hora de escoger los temas, los estudiantes revelaron su preocupación por abordar temas que les ayudaran a mejorar su competencia intercultural.

Para la redacción de los textos que se publicarían, los estudiantes se organizaron en grupos de tres y se hizo trabajo cooperativo en el que investigaron sobre los temas escogidos. En cada grupo se llevaron a cabo discusiones en las cuales los estudiantes definieron las ideas que se tratarían, escribieron los textos, se corrigieron entre sí para mejorarlos mediante la negociación de forma y significado y, finalmente, llegaron a acuerdos sobre la información que se publicaría.

\section{Análisis de los datos y resultados}

Con el fin de resolver la pregunta de investigación (¿Qué posible impacto puede tener la Web 2.0 en el desarrollo profesional de un grupo de docentes de inglés en formación?), se examinaron los datos de las entrevistas, las cuales fueron grabadas, así como información recogida en los blogs, podcasts y microblogs de los estudiantes y los datos de los pre y postests. Para este análisis se siguió el procedimiento de codificación abierta -open coding- (Strauss y
Corbin, 1990), el cual permite que el investigador lea, examine, rotule, compare, conceptualice y categorice la información con miras a identificar conceptos e información específica.

Las respuestas o comentarios de los participantes en todos los instrumentos se digitaron y agruparon por pregunta; luego se catalogaron respecto a tres ejes o categorías: componente tecnológico, componente pedagógico y componente lingüístico. Estas categorías fueron divididas a su vez en subcategorías, una vez codificados todos los datos por temas similares. A continuación presentaremos los resultados del análisis, ilustrando con evidencias tomadas de diversos instrumentos. Sin embargo, es necesario aclarar que aunque existe gran número de evidencias tomadas de cada uno de los instrumentos, solo presentaremos algunas dado el límite de espacio en este artículo.

Tabla 1. Categorías y subcategorías.

\begin{tabular}{|c|c|}
\hline Categorías & Subcategorías \\
\hline $\begin{array}{c}\text { Componente } \\
\text { tecnológico }\end{array}$ & $\begin{array}{c}\text { Conocimiento de las herramientas } \\
\text { Aplicación de las herramientas }\end{array}$ \\
\hline & \begin{tabular}{c} 
Cambio de actitud y percepción \\
\hline Herramienta de motivación e innovación
\end{tabular} \\
\hline Componente & $\begin{array}{r}\text { Herramienta de aprendizaje y práctica de la } \\
\text { lengua y de la cultura }\end{array}$ \\
\hline pedagógico & $\begin{array}{c}\text { Herramienta de construcción social de } \\
\text { conocimiento y de trabajo colaborativo }\end{array}$ \\
\hline Componente & $\begin{array}{r}\text { Herramienta que promueve } \\
\text { autonomía }\end{array}$ \\
\hline lingüístico & Mejora en la gramática \\
\hline & Mejora en las habilidades \\
\hline
\end{tabular}

\section{Categoría 1: componente tecnológico}

Los datos de esta categoría se agrupan a su vez en tres subcategorías.

\section{Subcategoría 1: conocimiento de las herramientas}

Los estudiantes participantes reconocieron haber conocido o mejorado su conocimiento de tales herramientas durante el curso. Como lo expresan algunos, no sabían de la existencia de algunas herramientas. 
No sabía que existían, y nunca me había molestado en usarlas. (Entrevista, pregunta 2, estudiante $2^{3}$ )

Sí (considero que hubo algún cambio en mi conocimiento y/o habilidad en el uso de las tecnologías de la información y la comunicación durante este semestre) ya que ignoraba muchas de las herramientas que utilizamos durante el semestre y mejoraron mis conocimientos. (Entrevista, pregunta 5 , estudiante 14 )

Se han hecho conscientes de que así como ellos no conocían las herramientas, puede haber docentes que no las conozcan y les aconsejan que se pongan al día en las nuevas tecnologías:

For that reason it is mandatory to know about technological uses, if not how we can keep students in touch with those tools if we do not know them, it will be almost impossible. A piece of advice for teachers "please, be up to date". (Blog, estudiante 15)

\section{Subcategoría 2: aplicación de las herramientas}

Los estudiantes no solo aprendieron sobre la existencia de las herramientas sino que también aprendieron a usarlas y a aplicarlas en su quehacer, puesto que aunque todos son docentes en formación, algunos ya laboran en instituciones de educación no formal.

Fue una gran experiencia porque en este momento hago uso de ellas en mi labor docente. (Entrevista, pregunta 3, estudiante 13)

El uso de las tecnologías para mí era muy limitado, pero este semestre pude no solo conocer, sin también utilizar y tener la oportunidad de crear podcasts y wikis. (Entrevista, pregunta 7 , estudiante 8)

Con esta experiencia no solamente se logró que los estudiantes aprendieran a utilizar las herramientas para su uso personal, sino que, como el MEN se lo ha propuesto, también que las integraran a sus

3 Con el propósito de mantener en la confidencialidad la identidad de los estudiantes, a cada uno de ellos se le asignó un número, el cual lo identificará en cada uno de los datos que tomaremos como evidencia en el análisis. prácticas como docentes de cualquier disciplina o lo hagan en su futuro quehacer:

Sí, ahora manejo esas herramientas y trato de apropiarlas para mi práctica. (Entrevista, pregunta 9, estudiante 9)

Es una manera de acercarse más a las generaciones más jóvenes y más prestas a los avances tecnológicos. (Entrevista, pregunta 11, estudiante 7)

\section{Subcategoría 3: cambio de actitud y percepción sobre las herramientas}

El curso complementado con la Web 2.0 permitió a los estudiantes conocer y usar las nuevas herramientas y, al mismo tiempo, les ayudó a cambiar la percepción y la valoración en relación con las TIC como herramientas pedagógicas, lo cual se ha podido ver en que han superado los prejuicios y prevenciones que sentían hacia dichas herramientas:

Hay un cambio de conciencia y percepción de estas herramientas; esto se debe precisamente a lo que se empieza a conocer y fortalecer. (Entrevista, pregunta 7 , estudiante 10 )

Antes de tener contacto con las TIC, creía que las herramientas que tiene un profesor se limitaban al tablero y al marcador, pero ahora veo que no solo son útiles sino llamativas, especialmente en la enseñanza. (Entrevista, pregunta 8, estudiante 8)

Antes de trabajar estas herramientas en la clase, yo no veía cómo podrían ser útiles a la hora de planear y ejecutar una clase. (Entrevista, pregunta 5 , estudiante 14)

Los estudiantes reconocen que las nuevas herramientas pueden ser útiles y que al usarlas se pierde el miedo que se tiene a enfrentarse a la tecnología. Este temor, que se notó en algunos estudiantes que no cumplieron con todas las actividades propuestas, en las cuales debían usar wikis, podcasts, Twitter o blogs, puede ser parte de la falta de seguridad que siente frente a lo desconocido, pues la tecnología, para muchos, es eso, algo desconocido.

(Me siento) muy bien usando la Web 2.0, creo que a veces no tenemos un contacto con estas 
herramientas porque nos da miedo enfrentarnos a la tecnología, pero cuando lo hacemos, vemos cuán útil puede ser para nuestras vidas. (Entrevista, pregunta 3 , estudiante 8 )

El mundo es cada vez más tecnológico y los niños y nuevas generaciones tienen mayor conocimiento de estos avances. Por esto es importante que el maestro no se estanque sino que se mueva al ritmo del mundo también. (Entrevista, pregunta 12 estudiante 5)

En los ejemplos anteriores evidenciamos que con esta propuesta se logró un cambio de actitud en los estudiantes, dado que reconocen la utilidad de las nuevas herramientas y la transformación de los procesos de enseñanza-aprendizaje de las lenguas, la cual requiere de un docente diestro en el diseño y conocimiento del uso eficiente de la tecnología. Veamos lo que dicen dos estudiantes en su podcast y microblog, respectivamente:

We can say that the role of the teacher is changing, and he or she must adapt to the new tools that are arriving, because the education of the new students depends on these new tools: (podcast, estudiante 14)

Our role as teachers of the new era is be a guide who review and direct the students' learning by using technological and interactive tools. (Sic, Twitter, estudiante 10)

Hablando de transformación de los procesos de enseñanza-aprendizaje, en estos últimos ejemplos se evidencia que los estudiantes son conscientes del cambio de rol del docente, quien ya no será el centro del proceso de enseñanza-aprendizaje sino que guiará al estudiante, quien será el eje del proceso con el uso de la tecnología.

\section{Categoría 2: componente pedagógico}

Con los datos dados por los estudiantes podemos evidenciar que ellos consideran que las herramientas sí son útiles a la hora de aprender y enseñar la lengua. Ellos expresaron que durante el curso pudieron ver la utilidad de la Web 2.0 como herramienta pedagógica que potencia la motivación y la innovación en el aprendizaje, que permite aprender la lengua y practicarla, que apoya la construcción del conocimiento y fomenta el trabajo en equipo y que promueve la autonomía.

\section{Subcategoría 1: herramienta de motivación e innovación}

Los estudiantes que participaron en todas las actividades planeadas, en las que se utilizaron las herramientas de la Web 2.0, lo hicieron con entusiasmo y según ellos lo expresan, motivados hacia el aprendizaje mismo de la lengua y hacia el uso de las herramientas.

Son medios que ayudan a incentivar el interés por el aprendizaje. (Entrevista, pregunta 3, estudiante 2)

The high power of teaching through the Internet and computers is based on motivation. If we can show them how to learn from a game, a forum or a chat, the students will realize that school can be fun and that they can learn while they are having fun. (Podcast, estudiante 14)

Esa motivación pudo haber sido causada por varias razones; por ejemplo, el hecho de ver sus trabajos publicados en la red y la posibilidad de ser leídos por otras personas, les permitió ver que el trabajo no era solo para su profesor, sino que otras personas podrían acceder a ellos y comentarlos. Como afirma Warschauer (2000, p.52), el estudiante se siente motivado cuando encuentra que el propósito de las actividades es relevante social y culturalmente, lo cual se logra con la tecnología.

Es interesante el ver que mi trabajo publicado fuera útil para otras personas que no conocía, ya que personas ajenas a mí comentaron mi texto. (Entrevista, pregunta 5, estudiante 6)

Otra razón puede ser, de acuerdo con Dornyei (2001), que los estudiantes se sienten motivados debido a que las herramientas aumentan la autoestima y mejoran la autoconfianza.

Fue motivador debido a que inspira a trabajar más duro e inclusive a atreverse a seguir fortaleciendo el uso académico de una forma más activa. (Entrevista, pregunta 4 , estudiante 7 ) 
Otra razón de la motivación puede venir del hecho que los estudiantes pueden acceder a la información que desean, según sus intereses y sin presión del tiempo, y a que trabajan a su propio ritmo.

I think that new technologies are great, because everybody can choose those kind of topics which they feel more interested about. Also, you don't have the pressure of the time, you can work on your own, and you have access to many interesting articles and books at the same time. (Sic, blog, estudiante 9)

No solo expresan los estudiantes que el uso de las TIC es motivador para ellos, sino que también podría serlo para los demás estudiantes. Esto nos permite que ver que probablemente, en el futuro, estos profesores usarán las herramientas con sus estudiantes.

Creo que utilizarlos para la academia sería algo motivador e innovador. (Entrevista, pregunta 3, estudiante 9)

...este curso me permitió ver cómo se puede sacar provecho de estas herramientas en el ámbito pedagógico para desarrollar una clase de manera diferente y así motivar a los estudiantes en la clase y mostrarles otras formas de usarlas. (Entrevista, pregunta 5, estudiante 14)

De la misma manera, los estudiantes opinaron sobre la innovación que traen consigo las herramientas mediadas por TIC. Ellos afirmaron que con el uso de las herramientas, el aprendizaje se hace menos monótono y se innova el proceso de enseñanza-aprendizaje:

Por supuesto... uno encuentra potencialidades para desarrollar nuevas propuestas didácticas a través del uso de la web. (Entrevista, pregunta 8, estudiante 15)

Me pareció un trabajo interesante, ya que el aprendizaje no se volvió monótono. (Entrevista, pregunta 4 , estudiante 2)

Además, son conscientes de la necesidad de que la tecnología sea utilizada para encontrar nuevas estrategias de enseñanza:
Teachers need to use Technology as a tool in order to find new strategies for teaching. (Twitter, estudiante 18)

\section{Subcategoría 2: herramienta de aprendizaje y práctica de la lengua y de la cultura}

Los estudiantes consideran las herramientas como facilitadoras del aprendizaje y de la práctica de la lengua en todos sus aspectos: comprensión de escucha, expresión oral, comprensión de lectura y expresión escrita, así como gramática:

Hubo práctica constante de las habilidades en producción oral y escrita. (Entrevista, estudiante 12 , pregunta 6)

Me pareció un trabajo excelente, el cual motivó a la mayoría de compañeros a esforzarse más y a mejorar las distintas habilidades comunicativas. (Entrevista, pregunta 4, estudiante 11)

Sí, puede afianzar las estructuras gramaticales en la lengua. (Entrevista, pregunta 7, estudiante18)

Todas las actividades que se llevaron a cabo requerían que los estudiantes se comunicaran publicando sus producciones de manera oral o escrita. Esto seguramente contribuyó a que las habilidades de la lengua se usaran, se practicaran y se mejoraran de manera integrada, dado que se requería de la lectura para poder expresar luego los puntos de vista, opiniones, etc. Para los estudiantes, el uso apropiado de las nuevas herramientas permite la construcción de un aprendizaje significativo. Esto se logra, según Norton y Wiburg (1998), debido a que las tecnologías integradas a las experiencias de aprendizaje ayudan al estudiante a resolver problemas reales y a participar en comunidades de conocimiento, todo debido a la diversidad de herramientas mediadas por el ordenador, el cual facilita el acceso a recursos auténticos.

Sí (las herramientas tecnológicas pueden ser útiles). Si se hace de la forma correcta, los niños y adultos pueden aprender de manera más significativa. (Entrevista, pregunta 11, estudiante 8)

Definitivamente son un medio que facilita la comunicación y que a la vez permite un aprendizaje 
más productivo, de la mano de un afianzamiento y seguridad sobre lo que se está aprendiendo. (Entrevista, pregunta 3, estudiante 7)

Another advantage of the Internet is that it offers the possibility to study online and share pedagogical knowledge. (Podcast, estudiante 2)

El ejemplo anterior nos permite ver que además de que los estudiantes pueden utilizar la red para participar de diversos tipos de comunidades del conocimiento, también se puede utilizar para compartir información pedagógica, es decir, desde el punto de vista del docente. Por otra parte, la construcción del aprendizaje significativo se dio fácilmente debido a que hubo posibilidad de interacción entre estudiantes y otros usuarios de las herramientas de la Web 2.0:

(El uso de la Web 2.0 tuvo incidencia en mi conocimiento) sobre todo en el proceso de interacción con otros y que a su vez facilitó procesos de identificación de elementos propios de la cultura extranjera. (Entrevista, pregunta 6, estudiante 7)

Como vemos en el comentario anterior, también se manifiesta que las herramientas de la Web 2.0 permiten el acceso a y el conocimiento de otras culturas, lo cual da lugar al mejoramiento de la competencia intercultural del individuo. Esto también se puede evidenciar en el siguiente ejemplo:

It is certainly true that interacting with people from other cultures, they open their minds to new concepts and styles. (Blog, estudiante 5)

\section{Subcategoría 3: herramienta de construcción social de conocimiento y de trabajo colaborativo} Para Butler-Pascoe (en Butler- Pascoe y Wiburg, 2003, p. 2), mediante el uso de la red el estudiante se puede sentir parte de una comunidad más grande que la del aula de clase, en la cual unos aprenden de otros, lo cual se evidenció en los datos de los estudiantes:

Uno se siente parte de algo importante. Es una colectividad en la red, distinta a la de hacer amigos y jugar videojuegos. Es una colectividad académica. (Entrevista, estudiante 10, pregunta 5)
Impacto de un curso mediado por la web 2.0 en el desarrollo

profesional de un grupo de futuros docentes de inglés

Me sentí muy bien aportando material para el aprendizaje de la lengua de otras personas. (Entrevista, pregunta 5, estudiante 13 )

The most wonderful fact that these tools offered is the chance of the cooperative learning method as a way to interact and to gain knowledge from the others and to the others. This is a very important aspect to have into account when using ITC, as teachers or as students. (Blog, estudiante 12)

A través de la interacción que se dio entre los estudiantes, particularmente en la wiki, hubo procesos de negociación de significado, de atención a aspectos formales de la lengua y de co-construcción del significado.

No tenía idea de la posibilidad de crear un wiki en el cual se construyera conocimiento de forma más estable y participativa. (Entrevista, pregunta 7, estudiante 7)

El trabajo en equipo se potenció, reafirmando nuestra esencia de seres sociales y participativos de un proceso evolutivo de aprendizaje. (Entrevista, pregunta 10 , estudiante 7 )

Los estudiantes tuvieron la oportunidad de corregirse unos a otros con el fin de llegar a negociación de contenidos y significados. Esto, según ellos, fue positivo porque vieron las correcciones de sus compañeros como algo constructivo y se dio dentro de una relación de respeto:

Fue gratificante porque fue un trabajo bien elaborado el que se publicó. Además, mediante esto nuestros compañeros pudieron hacer sus comentarios y críticas constructivas. (Entrevista, pregunta 5 , estudiante 4 )

Se evidenció el respeto que cada uno de nosotros puede manifestar frente a las opiniones y trabajo de los demás. (Entrevista, pregunta 10, estudiante 5)

El trabajo con la Web 2.0 ofreció la posibilidad de que los estudiantes utilizaran la lengua de forma interaccional -otros estudiantes y profesor- y transaccional -para desarrollar habilidades de la lengua y construir conocimiento-, las cuales son esenciales 
para una participación efectiva en el aula de clase (Butler- Pascoe y Wiburg, 2003, p.94).

El trabajo realizado con la Web 2.0 en el aula nos permitió interactuar de manera diferente a la habitual. Durante este proceso, nosotros nos ayudamos entre sí para mejorar nuestro nivel de lengua; hacíamos comentarios constructivos sobre el trabajo y el proceso de cada uno de los estudiantes y esto me parece útil, ya que tenemos una opinión diferente sobre nuestro trabajo en la clase. (Entrevista, pregunta 7, estudiante 14)

Fue gratificante ver mi trabajo en la web porque me permitió mejorar mis habilidades comunicativas y, además, esto permitió que mis compañeros vieran mi trabajo y pudieran hacer algún comentario o corrección que me permitieran mejorar mi nivel de lengua... hubo coevaluación. (Entrevista, pregunta 4, estudiante 14)

En el ejemplo siguiente se muestra que la red no solo permite acceder a herramientas interactivas, sino que también permite que el estudiante, el usuario o el docente creen sus propias actividades que promuevan el aprendizaje:

The Internet offers the possibility to ... create interactive tools that help students learn in a suitable way. (Podcast, estudiante 2)

\section{Subcategoría 4: herramienta que promueve autonomía}

El análisis de la información permitió evidenciar que los estudiantes reconocen que el trabajo de aula, complementado con el uso de herramientas de la Web 2.0, les dio la posibilidad de mejorar el trabajo autónomo y aumentar la autorregulación del aprendizaje:

Son fundamentales para crear un ambiente de aprendizaje con varias herramientas que motivan los estudiantes a desarrollar el trabajo autónomo y el amor por la cultura y lengua extranjeras. (Entrevista, pregunta 11, estudiante 1)

Ayudan al estudiante a autorregularse y autocorregirse. (Entrevista, pregunta 11 estudiante 9)
Cambió la forma de aprender. En mi caso, me enseñó a aprender a aprender, valga la redundancia, de múltiples maneras. (Entrevista, pregunta7, estudiante 2).

This new tools are good for learning... As students, we should use the best way possible this technological advances and take advantage of them; that means that we will also improve nor only our abilities but our autonomous work. (Sic, blog, estudiante 8)

Como parte del trabajo autónomo, los estudiantes son conscientes de que las nuevas herramientas les dan otras posibilidades de técnicas de estudio y de que su rol como estudiantes ha cambiado, pues ya no dependen del docente para mejorar su aprendizaje:

Hubo un mejoramiento en las técnicas de estudio utilizadas, y eso provoca un conocimiento mejor estructurado. (Entrevista, estudiante 13, pregunta7)

Ahora busco podcasts para practicar mi listening, en el cual considero tengo muchos problemas y antes no sabía cómo mejorar este aspecto sin la presencia de un profesor. (Entrevista, estudiante 8 , pregunta 8 )

Not only the role of the teacher has changed but also the role of the student, every day the student can find new challenges across the Internet and the different multimedia channels. (Blog, estudiante 14)

Ser consciente de las estrategias de aprendizaje hace al estudiante más autónomo; también, estas estrategias se potencian mediante el uso de los recursos tecnológicos, como lo manifiesta el estudiante que expresa:

When students start to work by themselves with the help of technological resources, they become more conscious about their own strategies of learning and develop more possibilities of research and problem situations. Their sense of responsability increases and they undertake the adventure of acting autonomously. They can go to a suitable rhythm for them, according to their time and needs. (Sic, blog, estudiante 5) 


\section{Categoría 3: componente lingüístico}

Para llegar a esta categoría se tuvieron en cuenta los errores más comunes que los estudiantes cometieron en los pretests oral y escrito y los postests, también oral y escrito, y la entrevista final que se les administró.

\section{Errores comunes de los estudiantes antes de la implementación}

Con el fin de analizar los datos se elaboró un inventario de errores de las composiciones de la siguiente manera: se leyeron una a una las composiciones y se numeraron. Luego, en la misma hoja de las composiciones se elaboró un listado indicando en qué consistía cada error. Por otro lado, el inventario de errores de la actividad oral se hizo tomando nota de los errores que se habían cometido durante cada una de sus intervenciones $y$, al igual que con las composiciones, se numeraron. En la figura 2 se presentan los aspectos en los cuales se han encontrado los errores más comunes cometidos por los estudiantes antes de la implementación del curso apoyado en la Web 2.0:

Figura 2. Errores del pretest

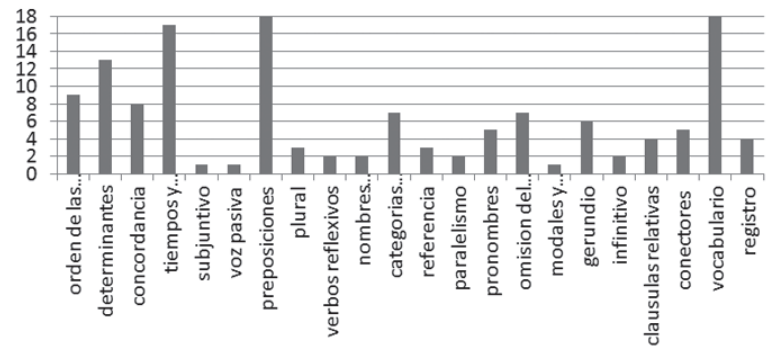

La mayoría de los errores se presentaron en el vocabulario, ya sea por no encontrar el término adecuado en ese momento o por confundir palabras que en español pueden tener significados similares; también en las preposiciones de lugar, de tiempo, o las que acompañan los verbos compuestos. El tercer aspecto de mayor frecuencia de error es el de tiempos verbales y conjugación y el cuarto es el del orden de las palabras dentro de la estructura oracional.

Los aspectos que presentan menor número de errores son el subjuntivo, la voz pasiva, los modales y auxiliares, seguidos de los verbos reflexivos, la diferencia entre contables y no contables y el infinitivo. Errores como los del subjuntivo son típicos del nivel en el que se encuentran los estudiantes y aún de estudiantes que poseen un muy alto nivel de manejo de la lengua inglesa.

\section{Subcategoría 1: mejora en la gramática}

El primero de los postests consistió en escribir una composición libre sobre algunos de los temas que los estudiantes habían escogido para la wiki, o sobre sus metas después de terminar los estudios, temática similar a la del pretest. El segundo consistió en una actividad similar a la del pretest: para tomar los datos del postest escrito se hizo, al igual que para el pretest, un inventario de los errores cometidos por cada alumno, y para los datos del postest oral, al escuchar a cada estudiante en sus intervenciones, se tomaba nota de cada uno de sus errores. La gráfica siguiente nos muestra los datos concernientes a los aspectos en los cuales los estudiantes cometieron errores y sus frecuencias:

Figura 3. Errores postest

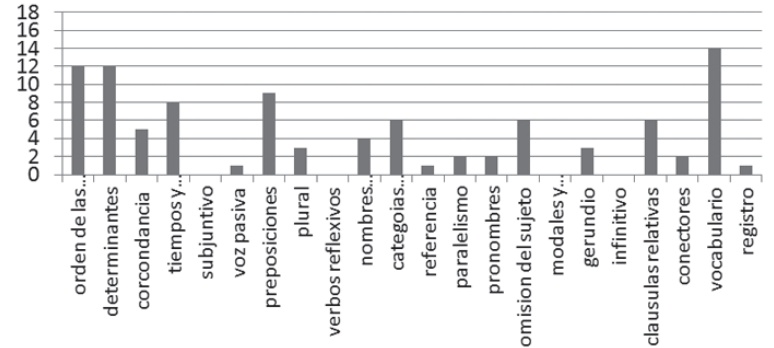

Al comparar las frecuencias de las gráficas correspondientes al pretest y al postest, podemos afirmar lo siguiente:

La competencia lingüística de los estudiantes no mejoró; por el contrario, se evidenció un aumento de la frecuencia de los errores en los siguientes aspectos:

- El orden de las palabras. Muchos de los casos detectados se presentaron en el uso del discurso indirecto.

- Diferencia entre nombres contables y no contables.

- Cláusulas relativas. 
El número de errores relacionado con la voz pasiva, paralelismo y formas del plural permaneció inalterado.

El desempeño de los estudiantes mejoró, aunque no de manera significativa, en los siguientes aspectos:

- El uso de determinantes. La mayoría de los casos que se presentaron en el postest tuvieron que ver con el uso del artículo determinado the.

- La selección apropiada de categorías morfosintácticas.

- Omisión de sujeto, especialmente del impersonal it, aunque se dieron casos de omisión de otros sujetos.

Finalmente, el nivel de los discentes mejoró sustancialmente en lo concerniente a:

- La concordancia sujeto-verbo.

- Tiempos verbales y conjugación verbal.

- Subjuntivo.

- Preposiciones.

- Verbos reflexivos.

- Relación referencia-referente.

- Pronombres.

- Modales y auxiliares.

- Gerundio.

- Infinitivo.

- Conectores.

- Vocabulario.

- Registro formal vs. Registro informal.

Así pues, a la luz de los datos desprendidos de los gráficos anteriormente presentados, se podría afirmar que los estudiantes mejoraron significativamente tanto en expresión oral como en expresión escrita, dado que de 22 temas, se mantuvo la frecuencia de errores o se aumentó tan solo en 6 de ellos. Por el contrario, la frecuencia de error disminuyó en los otros 16 aspectos. Esto se puede corroborar con la información que los estudiantes dieron en la entrevista final, la cual nos permite afirmar que hubo progreso en las diferentes habilidades y aspectos de la lengua debido al uso de las herramientas de la Web 2.0.

$\mathrm{Al}$ preguntar a los participantes sobre la incidencia del uso de herramientas de la Web 2.0 en su conocimiento y/o manejo de la lengua inglesa, los estudiantes reconocieron haber experimentado una mejoría en la estructura gramatical de la lengua inglesa. Es posible, como se puede ver en los ejemplos, que ello pudiera haber estado influenciado, en gran parte, por el papel de la retroalimentación que se dio durante la redacción de los textos a la hora de publicar en la red, hecho que no suele suceder en la redacción de forma manual.

Pude afianzar las estructuras gramaticales en la lengua. (Entrevista, pregunta 7, estudiante 18)

Sí, en gramática y escucha, sobretodo, ya que podía escribir los textos y recibir correcciones, para corregirlos varias veces y así (saber) cómo van las estructuras, si estaba usando una palabra equivocada, etc. (Entrevista, pregunta 6, estudiante 9)

\section{Subcategoría 2: mejora en el desarrollo de las habilidades}

$\mathrm{Al}$ responder la misma pregunta, los estudiantes afirmaron haber mejorado tanto su competencia oral como escrita, pero no la comprensión lectora, a pesar de haber trabajado con blogs y wikis, herramientas que exigen procesos simples y complejos de lectura por parte del usuario.

Creo que sí, pues con los podcast reforzamos la escucha y la producción oral, además la producción escrita, en la medida en que pudimos comentarlos, poner puntos de vista en los blogs y en las páginas sociales. También en la realización de wikis. (Entrevista, pregunta 5, estudiante 17)

Sí, incidió en el mejoramiento de habla y escritura. (Entrevista, pregunta 6, estudiante 13)

(Mejoré) las habilidades de speaking, listening, y writing porque al tener conocimiento de que otros tendrán acceso a esta información, tú te demandas más y trabajas un poco más para obtener un mejor resultado. (Entrevista, pregunta 6, estudiante 5)

\section{Subcategoría 3: mejora en vocabulario}

Uno de los aspectos con mayor frecuencia de error en el pretest fue el de vocabulario. No obstante, fue 
uno de los que representó mayor progreso por parte de los estudiantes, no solo como ellos lo comentan, en cuanto a vocabulario técnico relacionado con las tecnologías, sino también de acuerdo con las composiciones finales, en cuanto a vocabulario de diversas disciplinas.

Sobre todo en la adquisición de vocabulario técnico, ligado a las tecnologías. También se fortaleció el uso de conectores porque se trataba de hacer textos comprensibles para todos. (Entrevista, pregunta 6 , estudiante 16 )

Hay muchas palabras nuevas y un léxico propio que se maneja en la web... habilidades como el listening, writing y speaking se fortalecen. (Entrevista, pregunta 6 , estudiante 10 )

\section{Conclusiones}

Podemos concluir que el curso de inglés complementado por herramientas de la Web 2.0 ayudó a los participantes a mejorar su conocimiento de dichas herramientas, a considerarlas herramientas pedagógicas para apoyar el proceso de enseñanzaaprendizaje de la lengua y les ayudó a perfeccionar su dominio de la lengua hablada y escrita. Asimismo, tuvo un efecto positivo en el desarrollo profesional de los participantes en el aspecto tecnológico, dado que los estudiantes mejoraron su conocimiento y uso de las herramientas como estudiantes de la lengua y, como ellos lo manifiestan, también como docentes; porque se dio un cambio de actitud y de percepción frente a ellas: ahora las consideran herramientas pedagógicas que cumplen un rol en la educación actual y han perdido el temor de enfrentarse a ellas.

En el aspecto pedagógico, podemos afirmar que las herramientas de la Web 2.0 ayudaron a los estudiantes a aprender la lengua extranjera, porque a partir del trabajo realizado, ellos manifestaron haber aprendido a valorar la Web 2.0 como herramienta pedagógica que potencia la motivación en el estudiante, permite la innovación en el aprendizaje, se puede utilizar tanto para aprender como para practicar la lengua extranjera y conocer su cultura. También porque permite construir cono- cimiento significativo, ya sea de manera autónoma o colectiva y porque representa nuevas estrategias de aprendizaje. Esto se evidenció en que los 18 estudiantes que participaron en todo el proceso estuvieron motivados por desarrollar todas las actividades propuestas por la docente investigadora, en que algunos consideraron que las herramientas inyectaron variedad en el proceso de aprendizaje y afirmaron haber participado de trabajos colaborativos que les permitieron construir conocimiento de manera colectiva.

En lo tecnológico, los estudiantes mejoraron su conocimiento de algunas herramientas que no conocían o que habían oído mencionar, pero no habían utilizado ni como recursos de uso personal ni como recursos pedagógicos. Además, aprendieron a usar algunas herramientas, tales como microblogs, podcasts, blogs y wikis, con fines pedagógicos, pues las utilizaron para mejorar su aprendizaje como estudiantes de la lengua y, según la información recogida, manifestaron su intención de usarlas en el futuro como docentes. Por último, los estudiantes superaron su actitud reticente hacia el uso de las TIC, pues su utilización en el curso les permitió familiarizarse con ellas, perder el temor a usarlas, verlas como herramientas útiles.

En el aspecto lingüístico, se pudo evidenciar que el curso apoyado en la Web 2.0 tuvo incidencia positiva en el perfeccionamiento de la lengua hablada y escrita de los futuros docentes, dado que mejoraron tres de las cuatro habilidades de la lengua -comprensión de escucha, expresión oral y comprensión de lectura-, mejoraron el uso de la gramática en diversos aspectos y ampliaron su vocabulario sobre diversos temas, incluido el de la tecnología.

Podemos concluir que los efectos del curso apoyado en la Web 2.0 tuvieron un gran impacto en el desarrollo profesional de los estudiantes participantes, por cuanto tuvo incidencia en el conocimiento tecnológico, pedagógico y lingüístico de los estudiantes. Sin embargo, al igual que los estudiantes lo manifestaron en la entrevista, consideramos que las herramientas de TIC no reemplazarán al docente y que este seguirá siendo esencial en el proceso de enseñanza aprendizaje. 
En relación con la temática que se abordó en las discusiones y comentarios publicados en los blogs, los microblogs y los podcasts, las TIC, como herramientas pedagógicas, por ser novedosas para los estudiantes, considerando que antes en ningún curso las habían tratado, podemos afirmar que promovieron la participación de los estudiantes, sus deseos de investigar más al respecto y su disposición para reflexionar más sobre sus beneficios para la enseñanza y el aprendizaje de la lengua.

Finalmente, hubo algunas dificultades que se presentaron durante la implementación de esta investigación y fueron manifestadas por los estudiantes en la entrevista final. Las presentamos aquí con el fin de que en futuras investigaciones se puedan superar o minimizar: para algunos estudiantes, aprender a manejar las diversas herramientas tomó bastante tiempo debido a que no tenían muchos conocimientos al respecto. Por ello, sugieren se les dé mucha más información y más práctica con cada una de las herramientas antes de utilizarlas completamente, y trabajar solo una herramienta por curso y no varias, como se hizo en esta investigación. Para otros hubo difícil acceso a los equipos y a Internet desde su casa, por lo cual se buscó optimizar el uso de los recursos durante la clase. Asimismo, los estudiantes sugirieron trabajar blogs comunitarios para desarrollar más trabajo colaborativo, la creación de sus propios blogs en lugar de trabajar en el blog del docente y la posibilidad de trabajar temas libres que generen más controversia y que tengan más posibilidad de discusión.

\section{Referencias}

Abdous, M., Camarena, M. y Facer B. (2009). MALL Technology: Use of Academic Podcasting in the Foreign Language Classroom. ReCALL 21 (1), 76-95.

Anderson, P. (2007). What is Web 2.0? Ideas, Technologies and Implications for Education. JISC Technology and Standards Watch. Visitado el 1 de marzo de 2010

en http://www.jisc.ac.uk/whatwedo/services/techwatch/ reports/horizonscanning/

Barnes, K., Marateo, R. y Ferris, S. (2007). Teaching and Learning With the Net Generation. Innovate 3 (4).
Visitado el 13 de agosto de 2010 en http://www. innovateonline.info/index.php? view $=$ articleyid $=382$

Bautista, L.J., Martínez, R. y Sainz, I.M. (sin fecha). La evaluación de materiales didácticos para la educación a distancia. España: UNED. Visitado el 13 de agosto de 2010 en http://www.utpl.edu.ec/ried/images/pdfs/ vol4-1/evaluacion_materiales.pdf

Blood, R. (2000). Weblogs: A history and Perspective. En We've got Blog. Estados Unidos de América: Perseus Publishing.

Bloomberg, L.D. y Volpe, M. (2008).Completing your Qualitative Dissertation: A Roadmap from Beginning to End. Los Angeles, CA; London: Sage Publications.

Borau, K., Ullrich, C., Feng, J. y Shen, R. (2009). Microblogging for Language Learning: Using Twitter to Train Communicative and Cultural Competence. Visitado el 1 de marzo de 2010 en http://www.carstenullrich. net/pubs/Borau09Microblogging.pdf

Burns, A. (2003). Collaborative Action Research for English Language Teachers. Cambridge: CUP.

Burns, A. (2010). Doing Action Research in English Language Teaching. A Guide for Practitioners. New York: Routledge.

Butler-Pascoe, M. y Wiburg, K. (2003). Technology and Teaching English Language Learning. New York: A \& B.

Caballero, P., Prada, M., Vera, E. y Ramírez, J. (2007). Políticas y prácticas pedagógicas: las competencias en TIC en educación. Bogotá: Universidad Pedagógica Nacional.

Cabezas, C. (2008). Leer y escribir en la web social: uso de blogs, wikis y multimedia compartida en educación. Serie Bibliotecología y Gestión de Información, 35, 1-16.

Cabré, T. y Gómez de Enterría, J. (2006). La enseñanza de los lenguajes de especialidad: la simulación global. Madrid: Gredos.

Campbell, A.P. (2003). Weblogs for Use with ESL Classes. The Internet TESL Journal, v. 9, n. 2, Visitado el 3 de agosto de 2010 en: http://iteslj.org/Techniques/ Campbell-Weblogs.html

Campbell, D. y Stanley, J. (2001). Diseños experimentales y cuasiexperimentales en la investigación social. Buenos Aires: Amorrortu Editores.

Cantallops, E., González, R., y Yánez, E. (1999). Aprendizaje de lenguas europeas con ordenador e Internet. Nuevas perspectivas en la enseñanza del español como 
lengua extranjera I. Actas del X Congreso Internacional de ASELE. Cádiz: Universidad de Cádiz. 807-818.

Cantor, D. (2009). Discussion Boards as Tools in Blended, EFL Learning Programs. Profile. Issues in Teachers' profesional development, 11, 107-122.

Casanovas, M. (2002). Internet en la didáctica de las lenguas extranjeras: un paso adelante. Íkala. Revista de lenguaje y cultura, 7 (13), 53-60.

Chacón, C. y Pérez, C. (2009). El podcast como herramienta en la enseñanza del inglés como lengua extranjera. I Jornadas Virtuales sobre Conocimiento Libre y Educación CLED 2009. Universidad de Los Andes, Táchira, Venezuela. Visitado el 12 de marzo de 2010 en http://www.slideshare.net/carmentchacon/el-podcast-en-la-ensenanza-de-ingles

Chappelle, C. (2003). English Language Learning and Technology. Philadelphia: John

Benjamins Publishing Co.

Cunningham, K. (2000). Integrating CALL Into the Writing Curriculum. The Internet TESL Journal, VI (5). Visitado el 25 de febrero de 2010 en http://iteslj. org/Articles/Cunningham-CALLWriting/

Dave (Sin nombre). (2008). Twitter for Academia. Twitter by Dave.Visitado el 2 de junio de 2010 en http:// academhack.outsidethetext.com/home/2008/twitterfor-academia/

De la Torre, A. (2006). Web educativa 2.0. Edutec. Revista electrónica de tecnología educativa, 20. Visitado el 7 de julio de 2010 en http://www.uib.es/depart/gte/gte/ edutec-e/revelec20/anibal20.htm

Diaz-Maggioli, G. (2003). Professional Development for Language Teachers. ERIC Digest. EDO-FL 03-03. Agosto. Visitado el 9 de marzo de 2010 en http:// www.cal.org/resources/Digest/digest_pdfs/0303diaz. pdf

Dornyei, Z. (2001). Motivational Strategies in the Language Classroom. Cambridge: Cambridge University Press.

Edirisingha, P., Nie, M., Rizzi, C. y Rothwell, L. (2007). Podcasting to Provide Teaching and Learning Support for an Undergraduate Module on English Language and Communication.Turkish Online Journal of Distance Education-TOJDE. July ISSN 1302-6488, 3 (8).Visitado el 11 de mayo de 2010 en http://tojde. anadolu.edu.tr/tojde27/pdf/article_6.pdf.

Egbert, J. y Petrie, G. (2005). CALL Research Perspectives. USA: Lawrence Erlbaum Associates.
Freeman, D. (1998). Doing Teacher Research. From Inquiry to Understanding. Canada: Newbury House Teacher Development. Heinle \& Heinle.

Gimeno, A. y García, J. (2009). Wikis y el nuevo estudiante de lenguas extranjeras. Red U - Revista de Docencia Universitaria. Número Monográfico V. Número especial dedicado a Wiki y educación superior en España (II parte), en coedición con Revista de Educación a Distancia (RED).Visitado el 26 de julio de 2010 en http://www.um.es/ead/red/M12/7GimenoGarcia.pdf

Guzzo, E. (Sin fecha). La Web2.0 y las posibilidades didácticas para la enseñanza del español. (C.U. Newton Paiva/ ETFG- Sebrae- Mg). Visitado el 5 de julio de 2010 en http://www.letras.ufmg.br/espanhol/Anais/ anais_paginas\%20_2502-3078/La\%20Web\%20 2.0.pdf

Jonassen, D.H. (1996). Computers in the Classroom. Mindtools for Critical Thinking. Englewood Cliffs, New Jersey: Prentice Hall.

Jordano de la Torre, M. (2004). Blogging? Tell me How to Do That! V Jornadas Ceta. Córdoba. Facultad de Filosofía y Letras.

Jordano de la Torre, M. (2009). Promoting Communication and Autonomous Learning with the Help of Weblogs and RSS in an ESP Distance Education Context. XXVII Congreso Internacional de AESLA 2009, titulado "Ways and Modes of Human Comunication", March, 26th-28th.

Kessler, G. y Bikowski, D. (2010). Developing Collaborative Autonomous Learning Abilities in Computer Mediated Language Learning: Attention to Meaning Among Students in Wiki Space. Computer Assisted Language Learning, 23(1), 41.

Kitade, K. (2000). L2 Learners' Discourse and SLA Theories in CMC: Collaborative Interaction in Internet Chat. Computer Assisted Language Learning, 2, 143-166.

Lund, A. (2008). Wikis: A Collective Approach to Language Production. ReCALL, 20 (1),35-54. Visitado el 15 de mayo de 2010 en http://journals.cambridge. org/action/displayFulltext? type $=1$ yfid $=1584904 y j i d$ $=$ RECyvolumeId=20yissueId=01 yaid $=1584900$.

Marcelo, C. (2007). De la tiza al teclado: cambios, incertidumbres y aprendizaje en el proceso de convertirse en profesor online. Revista Interamericana de investigación, educación y pedagogía. 3, 1, 41-66. 
Merriam, S. (2000). Qualitative Research and Case Study Applications in Education. San Francisco: Jossey-Bass.

Ministerio de Educación Nacional. (2008). Ruta de desarrollo profesional docente para el uso de nuevas tecnologías. Apropiación de TIC en el desarrollo profesional docente. Bogotá. Visitado el 12 de diciembre de 2009 en http://www.iered.org/cmapserver/servlet/SBRead ResourceServlet?rid=1H1GMHSFS-1RHS5MF-1LQ

Ministerio de Educación Nacional. (2008). Revolución educativa. Plan sectorial 2006-2010. Documento No. 8. Visitado el 1 de diciembre de 2009 en

http://www.mineducacion.gov.co/cvn/1665/articles-2036_archivo_pdf.pdf

Norton, P. y Wiburg, K. (1998). Teaching with technology. Orlando: Hartcourt Brace y Co.

Osín, L. y Huergo J. (1999). Comunicación, humanismo y nuevas tecnologías en el espacio escolar. Bogotá: Universidad Pedagógica Nacional.

Pérez, M.I. (2004). Diseño de webquests para el aprendizaje del inglés como lengua extranjera. Tesis doctoral. Granada: Universidad de Nueva Granada.

Quintero. L.M. (2008). Blogging: A Way to Foster Writing. Colombian Applied Linguistics Journal, 10, 7-49.

Richardson, W. (2006). Blogs, Wikis, Podcasts, and Other Powerful Web Tools for Classrooms. Thousand Oaks, CA: Corwin Press.

Ruipérez, G., García, J., Castrillo, M. y Román, E. (2008). Aprender e investigar con Internet. Madrid: UNED

Stanley, G. (2006). Podcasting: Audio on the Internet Comes of Age. TESL-EJ on the Internet, 9 (4). Visitado el 20 de febrero de 2010 en http://tesl-ej.org/ ej36/int.pdf

Stevens, V. (2008). Trial by Twitter: The Rise and Slide of the Year's Most Viral Microblogging Platform.1 (12). Visitado el 18 de mayo de 2010 en http://tesl-ej.org/ ej45/int.pdf

Strauss, A. y Corbin, J. (1990). Basics of Qualitative Research Grounded Theory Procedures and Techniques. London: Seige Publications.

Vergara, O., Hernández, F. y Cárdenas, R. (2009). Classroom Research and Professional Development. Profile 11, 169-192.

Vygotsky, L.S. (1979). El desarrollo de los procesos psicológicos superiores. Buenos Aires: Grijalbo.

Warschauer, M. (1996). Motivational Aspects of Using Computers for Writing and Communication. In
M. Warschauer (Ed.), Telecollaboration in Foreign Language Learning: Proceedings of the Hawai'i Symposium. 29-46. Honolulu, HI: University of Hawai, Second Language Teaching and Curriculum Center.

Warschauer, M. (2000). On-line Learning in Second Language Classrooms: An Ethnographic Study. In M. Warschauer \& R. Kern (Eds.), Network-based Language Teaching: Concepts and Practice. 1-19. New York: Cambridge University Press.

Warschauer, M. y Grimes, D. (2007). Audience, Authorship, and Artifact: The Emergent Semiotics of Web 2.0. Annual Review of Applied Linguistics, 27, 1-23. Cambridge University Press.

West, J. y West, M. (2009). Using Wikis for Online Collaboration: The Power of the Read-write Web. San Francisco, CA: Jossey-Bass. 